\title{
Same, same, but different? Gelingende Kommunikation in und über Evaluation
}

\author{
Franziska Heinze, ${ }^{1}$ Stefanie Reiter, ${ }^{1}$ Stephanie Riedle ${ }^{2}$
}

\section{Idee einer handlungsfeldübergreifenden Session}

Die Evaluation komplexer (sozial-)politischer Programme stellt vielfältige Anforderungen an Evaluierende, da an der Entwicklung, Ausgestaltung und Umsetzung solcher Programme vielzählige Akteurinnen und Akteure aus unterschiedlichen Bereichen (z.B. Politik, Verwaltung, Wissenschaft, Kinder- und Jugendhilfe, Soziale Arbeit) beteiligt sind. Das Programm bildet den Interaktionskontext, in dem die beteiligten Akteurinnen und Akteure handeln und sich aufeinander beziehen (vgl. Heinze/Reiter/Berg-Lupper/Wach/Riedle 2019). Kommunikation spielt dabei eine zentrale Rolle: Sie ermöglicht den beteiligten Akteurinnen und Akteuren, sich einander verständlich zu machen, die jeweiligen Bedürfnisse $\mathrm{zu}$ artikulieren und die verschiedenen Positionen miteinander auszuhandeln. Dass dies gelingt, stellt jedoch keine Selbstverständlichkeit dar, denn die beteiligten Akteurinnen und Akteure sprechen mitunter sehr verschiedene ,Sprachen' und folgen unterschiedlichen Systemund Handlungslogiken. Kommunikative Herausforderungen werden dabei jedoch selten gemeinsam identifiziert, diskutiert und weiterbearbeitet.

Im Rahmen der 23. Jahrestagung der DeGEval - Gesellschaft für Evaluation e.V. zum Thema „Kommunikation: Verständigung und Sprache in der Evaluation“ $2020^{3}$ widmeten die Autorinnen diesem Spannungsfeld eine Session. Deren Ziel war es, Evaluierende sowie Projektumsetzende und Auftraggebende von Evaluationen bzw. Fördermittelgebende für Projekte mit ihren Erfahrungen und unterschiedlichen Perspektiven zusammenzuführen und miteinander ins Gespräch zu bringen. Gemeinsam sollten sie kommunikative Herausforderungen und Strategien zur Verständigung im Rahmen von (externen) Evaluationen diskutieren.

Die Initiatorinnen ${ }^{4}$ nahmen beispielhaft den (Interaktions-)Kontext von externen Evaluationen komplexer (sozial-)politischer Programme zum Ausgangspunkt

1 Deutsches Jugendinstitut e.V., Außenstelle Halle (Saale)

2 Deutsches Jugendinstitut e.V., München

3 Die Veranstaltung fand vom 17. bis 18. September 2020 als erste Online-Jahrestagung der DeGEval statt.

4 Die Session wurde von den drei Autorinnen des Beitrags initiiert und durchgeführt. 
der Session. Aus Sicht von Evaluierenden wurden einleitend zentrale Herausforderungen skizziert, die sich kommunikativ im Miteinander der Akteursgruppen bei der Umsetzung von externen Evaluationen u.a. von komplexen (sozial-)politischen Programmen stellen. Daran anknüpfend brachte die Session im Zuge einer interaktiven leitfadengestützten Fishbowl-Diskussion Expertinnen und Experten aus unterschiedlichen Bereichen (Evaluatorinnen und Evaluatoren, Programmfinanzierende und Projektumsetzende) zusammen und ins Gespräch zu Kommunikation in und über Evaluation. Im Fokus standen dabei die Aushandlung und Aushandlungsorte der verschiedenen Erwartungen, die Verständigung der Akteurinnen und Akteure zu den Zielen und zur Ausgestaltung von Evaluation sowie die weitere kommunikative Umsetzung von Evaluation. Hierbei wurden gezielt Erfahrungen aus deutschen und österreichischen Evaluationskontexten von drei Diskutantinnen bzw. Diskutanten vorgestellt, mit dem Publikum gemeinsam erörtert und um weitere Perspektiven bereichert. Ziel der Diskussion war herauszuarbeiten, welche kommunikationsbezogenen Aspekte ein gemeinsames Verstehen befördern oder verhindern und damit letztlich auch den Nutzen bzw. die Nutzung von Evaluation und ihrer Ergebnisse (Patton 2008; Fetterman/Rodríguez-Campos/Zukoski 2017).

Der Beitrag systematisiert die Beiträge der Session entlang der zentralen Diskussionslinien, benennt ausgewählte Erkenntnisse und offene Fragen. ${ }^{5}$

\section{2. (Kommunikative) Anforderungen in der Evaluation komplexer (sozial-)politischer Programme aus Sicht von Evaluierenden}

Die Session startete mit einem kurzen theoretischen Input von Stefanie Reiter zu zentralen Herausforderungen für Kommunikation in und über Evaluation. Ausgangspunkt waren dabei gemeinsame Überlegungen der Initiatorinnen der Session zu den unterschiedlichen Handlungslogiken der beteiligten Akteurinnen und Akteure und den sich daraus ergebenden sprachpragmatischen Erfordernissen bezüglich Verständigung, Übersetzen und Verstehen (Nerdinger 2011; Renn/Straub/Shimada 2002). ${ }^{6}$

Aus der einschlägigen Evaluationsliteratur sowie den Erfahrungen der Autorinnen aus der Evaluationspraxis ist bekannt, dass im Rahmen von externen Evaluationen - insbesondere bei komplexen Programmen - eine Vielzahl verschiedener Akteurinnen und Akteure aufeinandertreffen (z.B. Programmgebende, Projektumset-

5 Wir danken Alexandra Pertler (Deutsches Jugendinstitut e.V., Außenstelle Halle (Saale)) für ihre großartige Unterstützung bei der Dokumentation der Veranstaltung. Die Folien zur Session sind auf den offziellen Seiten der DeGEval-Tagungsdokumentation unter https://www.degeval.org/ver anstaltungen/jahrestagungen/online-tagung-2020/ veröffentlicht.

6 Im Vorfeld der Session erfolgte dazu ein intensiver, projektübergreifender Austausch zwischen externen Evaluierenden, die sich mit komplexen Programmen aus dem Bereich des Sozialen beschäftigten, und eine gemeinsame Erarbeitung von zentralen Rahmenbedingungen und Faktoren des Gelingens. Die Session wurde somit aus der Perspektive von Evaluierenden gestaltet und wollte diese Perspektive mithilfe des Einstiegs transparent machen. Sie hatte jedoch zum Ziel, im Gespräch mit den Expertinnen und Experten und dem Publikum diese Perspektive ein Stück weit aufzubrechen und das Thema aus verschiedenen Blickwinkeln - auch aus der projektumsetzenden Fachpraxis, von Programmgebenden etc. - zu beleuchten und vielleicht auch mit anderen Formen von Evaluationen oder aus anderen thematischen Bereichen und Kontexten zu kontrastieren. 
zende, Evaluierende). Diese sind häufig in unterschiedlichen Bezugssystemen (z.B. Politik, Verwaltung, Soziale Arbeit/Kinder- und Jugendhilfe/Schule, Wissenschaft/ Evaluation) verortet. Sie wirken im Rahmen eines (komplexen) Programms zusammen (vgl. Haubrich/Struhkamp/Lüders 2006; Widmer 2000). Das Programm als Evaluationsgegenstand wird durch diese Interaktion maßgeblich gestaltet oder sogar erst hervorgebracht. Innerhalb des gemeinsamen Interaktionskontextes nehmen die verschiedenen Akteurinnen und Akteure unterschiedliche Funktionen und Positionen ein. Zugleich ist ihr Handeln aufeinander bezogen, beispielsweise, wenn Projektumsetzende eine Förderung ihrer Vorhaben bei den Programmgebenden beantragen, wenn externe Evaluierende die Fachpraxis beforschen, um etwas über deren Sicht auf die Umsetzung von Maßnahmen zu erfahren oder wenn Stakeholder aus Politik und Verwaltung auf Evaluationsergebnisse zurückgreifen, um Nachsteuerungen und Verbesserungen in Programmumsetzungen einzuleiten. Für Evaluierende ergibt sich dabei oft die Herausforderung, ,zwischen den Stühlen' zu sitzen, also zu allen beteiligten Akteurinnen und Akteuren gleichermaßen tragfähige Arbeitsbeziehungen zu etablieren und deren Anliegen gleichzeitig zu berücksichten. Dies bringt zahlreiche, mitunter nicht immer gleichlaufende Anforderungen an ihr Handeln, an ihre Rolle/n mit sich (vgl. Heinze et al. 2019). Mit Blick auf die Kommunikation in und über Evaluation stellen sich dabei folgende Fragen:

- Welche zentralen Herausforderungen stellen sich kommunikativ im Miteinander der Akteurinnen und Akteure bei der Umsetzung von externen Evaluationen (von komplexen (sozial-)politischen Programmen)?

- Wie kann Kommunikation im Rahmen der Evaluation funktionieren und zu einer gelingenden Evaluation und Nutzung von Evaluation beitragen?

Insbesondere mit der Anforderung der Berücksichtigung verschiedener Gruppen Beteiligter in komplexen Programmen gehen aus Evaluationssicht einige zentrale Herausforderungen einher. Viele dieser Herausforderungen hängen mit den unterschiedlichen Bezugssystemen und Handlungslogiken der beteiligten Stakeholder zusammen: ${ }^{7}$

Die Akteurinnen und Akteure aus verschiedenen Bezugssystemen sprechen unterschiedliche ,Fachsprachen' (z.B. spezifische Formen von ,Politiksprache', ,Verwaltungssprache', ,Evaluationssprache ${ }^{`}$ oder auch eine ,sozialarbeiterisch geprägte Sprache'). Daraus ergibt sich im Miteinander die Herausforderung, verschiedene Sprachregister/-codes zu bedienen, und es werden Übersetzungsleistungen notwendig, um gegenseitiges Verstehen zu gewährleisten. Beispielsweise müssen Projektumsetzende ihre sozialarbeiterischen Vorhaben in eine Antrags- und Berichtssprache übersetzen, die Programmgebende aus Politik und Verwaltung verstehen. Evaluierende müssen in Erhebungen und Berichten das jeweilige Fachvokabular von Programmgebenden und Projektumsetzenden bedienen bzw. deuten können. Die Akteurinnen und Akteure folgen darüber hinaus unterschiedlichen Kommunikationskulturen und -konventionen. Während beispielsweise in sozialarbeiterischen Projekten oftmals die verbale Kommunikation überwiegt, sind Antrags- und Berichtsver-

7 An dieser Stelle sei darauf verweisen, dass insbesondere komplexe Programme mit einer Vielzahl unterschiedlicher Akteurinnen und Akteure verwoben sind, aber dass sich diese Herausforderung auch in anderen Kontexten stellen kann. 
fahren vor allem schriftlich organisiert. Daneben gibt es hierarchische bzw. nicht hierarchische, spontane bzw. strategische sowie transparente/öffentliche bzw. verdeckte Formen von Kommunikation. Diese gilt es für Evaluierende zu kennen, um angemessen in verschiedenen Kommunikationssettings agieren sowie verschiedene Kommunikationskonventionen berücksichtigen zu können. Dabei wirken sich Machtasymmetrien auf die Kommunikation aus. Beispielsweise sind im Rahmen von externen Evaluationen Programmgebende gleichzeitig Fördermittel- und Auftraggebende, externe Evaluierende ebenso wie die Projektumsetzenden Fördermittelempfangende. Zudem sind die Programmgebenden und Projektumsetzenden jeweils Teil des Evaluationsgegenstands und können ebenso wie die Evaluierenden Agenda-Setting betreiben. Darüber hinaus müssen die jeweiligen organisationalen und fachlichen Referenzsysteme von den Evaluierenden erkannt und kommunikativ berücksichtigt werden. Beispielsweise können die Projektumsetzenden sich primär als Dienstleistende im Bereich der Sozialen Arbeit verorten oder sich als Vertreterinnen und Vertreter einer zivilgesellschaftlichen Organisation verstehen, die staatliches Handeln, also auch das Handeln der Programmgebenden, kritisch überprüfen möchten. Dies zeigt, dass mit den unterschiedlichen Referenzrahmen auch unterschiedliche Funktionen einhergehen können, die für die Kommunikation in und über Evaluation bedeutsam sind.

Andere Autorinnen und Autoren formulieren diese Herausforderungen bezogen auf die Verständigung von Wissenschaft und Politik. Beispielsweise zeichnen sich für Heinrichs, Kropp und Wagner (2007) trotz systemtheoretisch begründeter Herausforderungen Verständigungsmöglichkeiten mit Blick auf die Wissenskommunikation im Dialog zwischen Wissenschaft und Politik ab: „So ist allgemein bekannt, dass die unterschiedlichen Zeithorizonte in Wissenschaft und Politik zu Schwierigkeiten bei der Synchronisation von Wissen und Entscheidungsfindung führen und ein von beiden Akteursgruppen diagnostiziertes Grundproblem darstellen. Hinzu kommen die unterschiedlichen Rationalitäten und Handlungszwänge von Wissenschaft und Politik, die alle Formen der Interaktion in gewisser Weise ,überschatten'. Jenseits dieser systemtheoretisch gut nachvollzogenen Probleme grundsätzlich verschiedener Orientierungen werden jedoch von den meisten Wissenschaftlern und Praktikern prinzipiell Verständigungsmöglichkeiten gesehen.“ (Heinrichs/Kropp/ Wagner 2007: 54).

Auch die Autorinnen betonen die Möglichkeiten zur kommunikativen Verständigung gegenüber den bestehenden Herausforderungen. ${ }^{8}$ Notwendig hierfür sind Orte und Wege der Aushandlung bzw. zur Förderung des gegenseitigen Verständnisses und Verstehens, welche je nach Setting, Anlage und Funktion der Evaluation gege-

8 Zielgruppenadäquat bzw. adressatengerecht zu kommunizieren gehört zu diesen Herausforderungen der Evaluationspraxis: Während Programmgebende oft kurze, steuerungsrelevante Rückmeldungen haben möchten, sind Projektumsetzende häufig primär an einer Reflexion ihres eigenen Tuns und an Vorschlägen zur Verbesserung interessiert. Darüber hinaus suchen alle Beteiligten immer auch Legitimation für ihr jeweiliges Handeln. Evaluierende benötigen dabei das Commitment aller beteiligten Akteurinnen und Akteure. Zudem sind sie gegebenenfalls in weitere Referenzsysteme (z.B. das Wissenschaftssystem oder die Konkurrenz zu anderen Evaluationsinstituten auf dem ,Evaluationsmarkt ${ }^{\star}$ ) eingebunden, die weitere sprachpragmatische Anforderungen mit sich bringen können. 
benenfalls erst gezielt geschaffen werden müssen (vgl. auch Heinze et al. 2019). Angesichts der Rollenförmigkeit der Interaktionsbeziehung sollte dabei bedacht werden, dass Kommunikation instrumentelles Handeln (was soll wie und zu welchem Zweck erreicht werden) bedeutet, zugleich aber immer auch soziales Handeln (Hacker 2009) umfasst und die Beziehungsebene im kommunikationspsychologischen Sinn definiert (Nerdinger 2007; Watzlawick/Beavin/Jackson 2017). In diesem Sinne kann adaptives Kommunikationsverhalten, also die situative Anpassung des Kommunikationsverhaltens unter Berücksichtigung der Sach- und Beziehungsebene, die instrumentellen (Kommunikations-)Handlungen maßgeblich unterstützen. Dabei spielen der Umgang mit Emotionen und die Etablierung einer förderlichen Sozialbeziehung eine wichtige Rolle (Nerdinger 2011). Eine zentrale Herausforderung für Evaluierende besteht somit darin, Vertrauen aufzubauen, multiple Erwartungen kommunikativ zu bedienen und sie so befriedigen, dass alle beteiligten Akteurinnen und Akteure einen Teil ihrer Erwartungen erfüllt bekommen. Dies kann die Basis für ein Verstehen bilden - und das gegenseitige Verstehen stellt nicht zuletzt eine grundlegende Voraussetzung für das Lernen aus Evaluationen und die Nutzung von Evalualtionsergebnissen dar.

\section{Verschiedene Perspektiven - gemeinsame Herausforderungen?}

Es folgte eine moderierte Fishbowl-Diskussion ${ }^{9}$ anhand konkreter Leitfragen mit Expertinnen und Experten, die in ihrer jeweiligen Rolle als Evaluierende, Programmfinanzierende und Programmumsetzende antworteten, und zugleich aus verschiedenen Bereichen (Verwaltung, Wissenschaft und Zivilgesellschaft) stammten. Als feste Diskutant(inn)en nahmen Magdalena Schwarz, Geschäftsführerin der österreichischen Bundes Jugend Vertretung (Wien), Christopher Gess, Referent im Referat für Evaluation, Internationales und Forschung zu Demokratie und Vielfalt im Bundesministerium für Familie, Senioren, Frauen und Jugend (Berlin) und Stephanie Riedle, wissenschaftliche Referentin in der Arbeitsstelle europäische Jugendpolitik am Deutschen Jugendinstitut e.V. (München) daran teil. Moderiert wurde die Session von Franziska Heinze. In der Fishbowl-Diskussion wurde aufseiten der Organisatorinnen auf mehrjährige Evaluationserfahrungen aus der Umsetzung der EUJugendstrategie in Deutschland und von Bundesprogrammen im Bereich der Demokratieförderung und Extremismusprävention zurückgegriffen. Weiterhin kamen Programmverantwortliche aus einem deutschen Bundesministerium sowie aus der österreichischen Bundes Jugend Vertretung zu Wort. Deren Vertreterin nahm zugleich auch eine Perpektive als Projektumsetzende ein. Die an der Session teilnehmenden Personen und deren Erfahrungen als Auftraggebende, Evaluierende oder Evaluierte wurden gezielt einbezogen.

9 Diese fand im Rahmen der Online-Tagung virtuell statt. Wie in einer Fishbowl-Diskussion üblich, gab es einen Innenkreis mit drei ,festen' Diskutantinnen bzw. Diskutanten und zwei freien, virtuellen' Stühlen. Die freien Stühle konnten wechselnd von Personen aus dem Außenkreis (Sessionteilnehmende) besetzt werden, d.h., wer sich einbringen wollte, konnte - virtuell leider nur gedanklich - einen der freien Stühle einnehmen und mitdiskutieren. 
Im Folgenden werden die Diskussionsbeiträge entlang der in der Fishbowl dominierenden Diskussionsstränge dargestellt.

\subsection{Kommunikation in Evaluationen als Prozess begreifen}

In der Diskussion wurde deutlich, dass Kommunikation sowie Aushandlungen über die gesamte Laufzeit einer Evaluation unabdingbar für das gegenseitige Verstehen und eine gelingende Evaluation sind.

Unter den Teilnehmenden herrschte Einigkeit darüber, dass bereits zu Beginn einer Evaluation eine Klärung offener Fragen aller beteiligten Akteurinnen und Akteure zu Zielen, zur Anlage und zur Umsetzung der Evaluation stattfinden sollte. Hierfür braucht es aufgrund der unterschiedlichen Sprachen der Beteiligten sowie der verschiedenen System- und Handlungslogiken, denen sie folgen, eine grundsätzliche Offenheit aller Beteiligten, sich auf entsprechende Kommunikationsprozesse im Rahmen der Evaluation einzulassen und Klärungsprozesse anzustoßen. Gerade auch im Kontext komplexer (sozial-)politischer Programme kommt den Evaluierenden hierbei die wichtige Aufgabe zu, entsprechende Klärungen voranzubringen. Dies erscheint nicht nur aus Evaluierendensicht sinnvoll; die Rekonstruktion von Bedarfen und Interessen durch Evaluierende sowie deren Rückspiegelung, beispielsweise an Auftraggebende, kann entsprechende Klärungen auf allen Seiten, und damit nicht zuletzt auch die Nutzung von Evaluation, befördern. Evaluierenden wird dabei das Potenzial zugeschrieben, methodisch geleitetetes Fremdverstehen zu ermöglichen.

Das gegenseitige Verstehen der beteiligten Akteurinnen und Akteure wird als eine der zentralen Herausforderungen in Evaluationsprozessen angesehen: Evaluationen sind sehr dynamisch und folgen einer eigenen Logik, die meist nicht offensichtlich und daher erklärungsbedürftig ist. Insbesondere aus Sicht von Programmgebenden und Projektumsetzenden erscheint es notwendig aufzuzeigen, was eine (externe) Evaluation zu leisten vermag und was nicht, worin das Ziel der Evaluation liegt und wie es erreicht werden soll. Zudem waren sich die Diskussionsteilnehmenden einig, dass Evaluierende wissen sollten, welche Erkenntnisse der Evaluation welche Stakeholder zu welchen Zeitpunkten benötigen. Dieses Wissen sollte nicht zuletzt bei der Gestaltung der Evaluation berücksichtigt werden. Maßgeblich für einen guten Gestaltungsprozess ist das wechselseitige Vertrauen der Akteurinnen und Akteure aus den unterschiedlichen Bereichen, aber auch ein grundsätzliches Vertrauen in das Evaluationsgeschehen. Dazu gehört auch die Bereitschaft, über Missverständnisse oder Fehler zu diskutieren, um den Verlauf einer Evaluation oder eventuell folgender zu verbessern. Letztendlich sind der Nutzen bzw. die Nutzung der Evaluation und ihrer Ergebnisse aufseiten der Stakeholder bedeutsam, um zu robustem, brauchbarem Wissen ${ }^{10} \mathrm{zu}$ kommen.

$10 \mathrm{Zu}$ robustem Wissen vgl. Weingart/Lentsch 2008: $50 \mathrm{f}$. 


\subsection{Rahmenbedingungen für Kommunikation in Evaluationen mitdenken}

Ein weiterer Diskussionsstrang entfaltete sich entlang der Rahmenbedingungen für Kommunikation in Evaluationen. Dabei standen die Einwerbung bzw. Beauftragung von Evaluationen, die Etablierung von Aushandlungsorten und die Evaluationskultur der ausführenden Institutionen im Fokus.

Angesprochen wurde, dass bereits im Vorfeld der Einwerbung bzw. Beauftragung von Evaluationen die Erwartungen der auftraggebenden und der auftragnehmenden Seiten geklärt und die jeweiligen Bedarfe verdeutlicht werden sollten. Damit kann erreicht werden, dass eine grundsätzliche Vorstellung von der späteren Evaluationsumsetzung vorhanden ist und der Erwartungshorizont kommunikativ abgesteckt wird. Zudem sollte bereits definiert werden, welche Stakeholder zu welchem Zeitpunkt in den Kommunikationsprozess in und über die Evaluation einbezogen werden. Mit Blick auf die Einwerbung und Beauftragung von Evaluationen wurde dabei als problematisch betrachtet, dass Antragsformulare bzw. Leistungsangebote häufig umfangreiche Konkretisierungen erfordern. Entsprechende kommunikative Festlegungen stehen hierbei nicht selten im Widerspruch zu Aushandlungserfordernissen während der Umsetzung von Evaluationen. Dies wurde insbesondere am Beispiel von Evaluationen komplexer (sozial-)politischer Programme besprochen, die durch dynamische Programmumsetzungen charakterisiert und unterschiedlichen Einflüssen ausgesetzt sind. Befürchtet wurde, dass die notwendige Flexibilität für Aushandlungsprozesse durch entsprechende Kommunikationsformate (beispielsweise starre Leistungsbeschreibungen) eingeschränkt ist. Für die Erstellung von Evaluationsangeboten bedeutet dies, sie so zu formulieren, dass bestimmte Stellen nicht bis ins Detail konkretisiert, sondern während der Umsetzung der Evaluation gemeinsam mit den beteiligten Akteurinnen und Akteuren ausgehandelt und geklärt werden. Eine grundsätzliche Offenheit des Angebots/Antrags für notwendige Änderungen, die es ermöglicht, ggf. situationsangemessen und -bedingt den Evaluationsprozess anzupassen, wird als ein Faktor für das Gelingen von Evaluationen und das Hervorbingen nutzbarer Ergebnisse gesehen.

Es ist wichtig, dass Aushandlungsorte geschaffen werden, sei es explizit und fortwährend in eigens dafür eingerichteten Aushandlungsorten (wie Gremien) oder in informellen Settings (z.B. ,Tür- und Angelgespräche'), um eine fortwährende Kommunikation zu gewährleisten. Inhalte des Austauschs mit allen beteiligten Akteurinnen und Akteuren sollten das Vorhaben, der Zweck und die Ziele, aber auch Grenzen, der Evaluation sein.

In Bezug auf die ausführenden Institutionen wird eine eigene Evaluationskultur als wichtig erachtet. Diese kann über hausinterne Schulungen, vor allem, wenn noch keine ausgeprägte organisationsinterne Evaluationskultur besteht, geschaffen werden. In der Kommunikation wird der Wissenstransfer zu Evaluation für wichtig erachtet. Dies reicht von der Vermittlung gängiger Begrifflichkeiten, über die angewandten Evaluationsdesigns und damit einhergehender Erfahrungen bis hin zum Umgang mit dem Budget. 


\subsection{Kommunikative Herausforderungen bearbeiten}

Von den Sessionteilnehmenden wurden zahlreiche Punkte angeführt, wie die Kommunikation in und über Evaluation an häufig bislang unzureichend bearbeiteten Herausforderungen verbessert werden kann. Besonders hervorgehoben wurde, dass es wichtig sei, die Fachsprache der Evaluierenden an die Sprachkonventionen der Adressatinnen und Adressaten bzw. Stakeholder anzupassen sowie deren kommunikative Gepflogenheiten zu berücksichtigen. Daneben wurde vorgeschlagen, im Sinne von ,ground work' grundlegendes Wissen über Evaluation an Stakeholder zu vermitteln. Diese Punkte liefern einerseits wertvolle Ansatzpunkte, um die Kommunikation in und über Evaluation zu verbessern. Zugleich wurden sie kontrovers diskutiert, da mit Fachsprachen und Fachwissen immer auch kommunikative Distinktionsmöglichkeiten verbunden sind, die nicht nur mit Blick auf die Professionalisierung des Berufsfeldes Evaluation, sondern auch in einem ,umkämpften Evaluationsmarkt‘ für Evaluationsdienstleistende von (durchaus existenzieller) Bedeutung sind.

Weitere Möglichkeiten zur Verbesserung der Kommunikation in und über Evaluation wurden aufseiten von Evaluierenden insbesondere darin gesehen, zu Beginn des Evaluationsprozesses eine umfassende Ausarbeitung und Darstellung des Nutzens der einzelnen Erhebungsschritte vorzunehmen. So können den Stakeholdern die Ziele und das Design der Evaluation verdeutlicht werden. Dies wird auch deshalb als wichtig angesehen, damit es den an der Evaluation beteiligten Akteurinnen und Akteuren ermöglicht wird, zu verstehen und in ihre eigenen ,Sprache c zu übersetzen, was das Ziel der Evaluation ist und wie dieses erreicht werden kann. Dadurch sollen Evaluationen in die Handlungslogiken aller beteiligten Akteurinnen und Akteure Eingang finden und die Legitimation der Evaluation in deren jeweiliger Arbeit erhöht werden.

Für eine gemeinsame Weiterarbeit aller am Evaluationsprozess beteiligten Akteurinnen und Akteure ist es zudem wichtig zu wissen, was die Evaluation leisten kann und wo sie an Grenzen stößt. In der Session wurde dafür plädiert, die Leistungspotenziale von Evaluationen transparent darzustellen, frei nach dem Credo ,Mut zur Kommunikation, Mut zur Ehrlichkeit‘. Die Diskussion verdeutlichte, dass es allen beteiligten Akteurinnen und Akteuren zuzumuten sei, den Evaluationsprozess authentisch und in seiner Komplexität darzulegen. Dabei trägt der (kontinuierliche) Austausch zu einem gegenseitigen Vertrauensverhältnis bei, ohne das eine Evaluation nur schwer gelingen kann. Angesichts der derzeitigen pandemischen Situation und den damit verbundenen Beschränkungen, auch für Evaluationen, stellt sich auch die Frage: Wie kann es gelingen, eine Vertrauensbasis mit den Stakeholdern zu etablieren, wenn Aushandlungsprozesse und die Zusammenarbeit im Kontext von Evaluationen primär im digitalen Raum stattfinden und notwendige informelle Gespräche wegfallen?

Last but not least wurde angemahnt, dass auch Programm- bzw. Auftraggebenden eine bedeutsame Rolle bei der Verbesserung der Kommunikation in und über Evaluation zukommt. Mit ihren budgetären Vorgaben, aber ebenso im Hinblick auf den transparenten Umgang mit Evaluationsergebnissen bzw. deren Veröffentlichung, 
tragen sie maßgeblich dazu bei, die Kommunikation in und über Evaluation zu verbessern.

\subsection{Kommunikation von Evaluationsergebnissen verbessern}

Als letzter und zugleich sehr bedeutsamer Punkt wurde in der Session die Frage der Verbesserung der Kommunikation von Evaluationsergebnissen thematisiert. Als eine Schwierigkeit wurde vonseiten der Diskutierenden benannt, dass Evaluierende häufig die (Kommunikations-)Bedürfnisse aller beteiligten Akteurinnen und Akteure gleichermaßen berücksichtigen müssen. Veranschaulicht wurde dies am Beispiel von ausführlichen Ergebnisberichten, die sich an Stakeholder aus unterschiedlichen Bereichen (z.B. Politik, Verwaltung, Projektpraxis und Wissenschaft) richten und damit vor der Herausforderung stehen, verschiedene Kommunikationskonventionen, Sprachregister und Nutzungsinteressen gleichzeitig zu bedienen - und diese damit fast zwangsläufig auch zumindest teilweise unbefriedigt zu lassen. Die Teilnehmenden der Session waren sich einig, dass diese - trotz der bekannten Schwierigkeiten - häufig geforderte Form der Ergebnisdarstellung eine mäßig geeignete Form der nutzungsorientierten Ergebniskommunikation ist.

Vorgeschlagen wurde unter anderem, bereits zu Beginn des Evaluationsprozesses verschiedene mündliche und schriftliche Formate der Ergebnispräsentation zu vereinbaren, um eine ,Übersetzung' der Evaluation und ihrer Ergebnisse zielgruppenspezifisch aufbereiten zu können. Als gängige und zielgruppenadäquate Kommunikationsformate für Politik, Verwaltung und Projektpraxis wurden Kurzberichte von bis zu 50 Seiten, Workshops und kombinierte Formate aus Präsentation und Diskussion genannt. Bei kurzfristigen Bedarfen werden Gesprächsformate (Briefings, Telefonate) und Kurzpapiere von 5 Seiten als sinnvoll erachtet. Zur Kommunikation von Ergebnissen in das Wissenschaftssystem wurden peer-reviewte Artikel angeführt. In der Summe - so formulierte es eine Teilnehmerin der Session - müssten Evaluationsergebnisse immer attraktiv, authentisch und in der ihrem Gegenstand angemessenen Komplexität dargeboten werden. Einigkeit unter den Sessionteilnehmenden herrschte darin, dass der, one size fits all'-Bericht als Format der Ergebniskommunikation ausgedient habe.

\section{Kommunikationskompetenz als Teil des Anforderungsprofils von Evaluierenden? - Fazit und Ausblick}

Bereits im Vorfeld der Online-Tagung ließ eine große Anzahl an Anmeldungen von Personen aus unterschiedlichen Bereichen ein hohes Interesse an den in dieser Session aufgeworfenen Fragen zum Thema Herausforderungen der Kommunikation in und über Evaluation erahnen. Die Teilnehmendenzahl von circa 90 Personen blieb auch während der Session konstant erhalten. Zahlreiche Wortbeiträge aus dem Innenkreis von den geladenen Expertinnen und Experten sowie aus dem Außenkreis 
vom Publikum verdeutlichten die Notwendigkeit des Austausches und der Verständigung zu diesem Thema und ergaben eine rege Diskussion.

Einige zentrale Punkte wurden im weiteren Tagungsverlauf unter Bezug auf die Session an unterschiedlichen Stellen immer wieder aufgegriffen. Hierzu gehörten die im einleitenden Input umrissenen multiplen Handlungsanforderungen an Evaluierende (vgl. grundlegend Heinze et al. 2019) und die Diskussion um angemessene Ergebnisformate vor dem Hintergrund der unterschiedlichen Kommunikationsbedürfnisse und -gewohnheiten der verschiedenen Adressatinnen und Adressaten. Deutlich wurde, dass Fragen der Kommunikation in und über Evaluation auch mit Blick auf das Anforderungsportfolio an Evaluierende aufgeworfen werden müssen. Denn Evaluierende sollten mindestens in der Lage sein, die verschiedenen Perspektiven der anderen an Evaluation beteiligten Akteurinnen und Akteure zu kennen und zu erfassen. Sie sollten die identifizierten Bedürfnisse der unterschiedlichen Stakeholder - gegebenenfalls unter Einbeziehung weiterer Expertise - bedienen können, z.B., indem sie zielgruppenadäquate Kommunikationsformate etablieren, die Sprachregister und -codes der jeweiligen Stakeholder kennen und deren Kommunikationskonventionen berücksichtigen. Daneben sollten Evaluierende daran mitwirken, geeignete Rahmenbedingungen für Kommunikation in und über Evaluation zu schaffen (z.B. Etablierung von Aushandlungsorten, Bereitstellen von Ressourcen für Kommunikation). Hierzu kann einerseits, ground work' einen Beitrag leisten, also die grundlegende Vermittlung von Wissen bezüglich Evaluation an Stakeholder sowie deren Aufklärung über Leistungspotenziale und Grenzen von Evaluation. Andererseits kann die Weiterentwicklung und Verbreitung einer Evaluationsfachsprache im Sinne eines Fachvokabulars, das als gemeinsame Basis des gesamten fachlichen Austauschs dient hierzu beitragen. Offen blieb die Frage, ob umfassende Expertise zur Aufbereitung von Ergebnissen zum notwendigen Qualifikationsportfolio von Evaluierenden zu zählen ist oder ob diese Expertise als zusätzliche Ressource für Evaluationen eingeplant und auch ein entsprechendes Budget bereitgestellt werden sollte.

Einige der spannenden Diskussionspunkte finden sich auch in den Fragen wieder, die der Call für die nächste DeGEval-Jahrestagung aufwirft: Wie müssen Empfehlungen formuliert sein, damit sie von den Auftraggebenden wahrgenommen und ggf. in eine Agenda aufgenommen werden? Wie haben sich in den vergangenen Jahren die Qualitätsanforderungen an Evaluationen und damit auch die Qualifikationsprofile der Evaluierenden geändert? ${ }^{11}$ Mit Spannung wird daher auch das Arbeitspapier der DeGEval-ad-hoc-Gruppe ,Revision der Empfehlungen für Aus- und Weiterbildung in der Evaluation' erwartet, welches eine Aktualisierung und Überarbeitung des Anforderungsprofils für Evaluatorinnen und Evaluatoren anstrebt. ${ }^{12}$ Somit werden diese Diskussionen auch in der DeGEval, als einer zentralen Plattform für Kommunikation in und über Evaluation, weitergeführt werden.

11 Der Call ist unter https://www.degeval.org/veranstaltungen/jahrestagungen/muenster-2021/call-forproposals/ einsehbar.

12 Informationen zu dieser Arbeitsgruppe sind unter https://www.degeval.org/arbeitskreise/ad-hocgruppen/ auffindbar. 


\section{Literatur}

Fetterman, David M./Rodríguez-Campos, Liliana/Zukoski, Ann P. (2017): Collaborative, Participatory, and Empowerment Evaluation. Stakeholder Involvement Approaches. New York: Guilford Press.

Hacker, Winfried (2009): Arbeitsgegenstand Mensch: Psychologie dialogischinteraktiver Erwerbsarbeit. Lengerich: Pabst.

Haubrich, Karin/Struhkamp, Gerlinde/Lüders, Christian (2006): Einleitung: Aushandlungsprozesse in externen Evaluationen. Aufarbeitung eines „stillen Themas“. In: Projekt eXe (Hg.): Wirkungsevaluation in der Kinder- und Jugendhilfe. Einblicke in die Evaluationspraxis. München: DJI, S. 5-18.

Heinrichs, Harald/Kropp, Cordula/Wagner, Jost (2007): Politische Meinungsbildung im Dialog von Wissenschaft und Politik. Eine sozialwissenschaftliche Reflexion der Erfolgsbedingungen anhand des Agrarbereichs. In: Kropp, Cordula/Schiller, Frank/Wagner, Jost (Hg.): Die Zukunft der Wissenskommunikation. Perspektiven für einen reflexiven Dialog von Wissenschaft und Politik - am Beispiel des Agrarbereichs. Berlin: Edition Sigma, S. 51-71. https://doi. org/10.5771/9783845267203-51

Heinze, Franziska/Reiter, Stefanie/Berg-Lupper, Ulrike/Wach, Katharina/Riedle, Stephanie (2019): Multiple Handlungsanforderungen an Evaluierende. Ein theoretisches und praktisches Problem in der Evaluation komplexer (sozial-)politischer Programme. In: Zeitschrift für Evaluation, 18 (2), S. 261-283. https://doi.org/10.31244/zfe.2019.02.05

Nerdinger, Friedemann W. (2007): Dienstleistung. In: von Rosenstiel, Lutz/Frey, Dieter (Hg.): Marktpsychologie. Enzyklopädie der Psychologie. Göttingen: Hogrefe, S. 375-418.

Nerdinger, Friedemann W. (2011): Psychologie der Dienstleistung. Bern: Hogrefe.

Patton, Michael Q. (2008): Utilization-Focused Evaluation. Thousand Oaks: Sage (4. Aufl.).

Renn, Joachim/Straub, Jürgen/Shimada, Shingo (Hg.) (2002): Übersetzung als Medium des Kulturverstehens und sozialer Integration. Frankfurt/New York: Campus Verlag.

Watzlawick, Paul/Beavin, Janet/Jackson, Don (2017): Menschliche Kommunikation: Formen, Störungen, Paradoxien. Bern: Hogrefe. https://doi.org/10.1024/85745-000

Weingart, Peter/Lentsch, Justus (2008): Wissen - Beraten - Entscheiden. Form und Funktion wissenschaftlicher Politikberatung in Deutschland. Weilerswist: Velbrück Wissenschaft.

Widmer, Thomas (2000): Qualität der Evaluation. Wenn Wissenschaft zur praktischen Kunst wird. In: Stockmann, Reinhard (Hg.): Evaluationsforschung. Grundlagen und ausgewählte Forschungsfelder. Wiesbaden: VS Verlag für Sozialwissenschaften, S. 77-102. https://doi.org/10.1007/978-3322-92229-8_4 